\title{
SUMMING A COMMON TYPE OF SLOWLY CONVERGENT SERIES OF POSITIVE TERMS
}

\author{
J. E. DRUMMOND
}

(Received 6 June 1976)

(Revised 30 September 1976)

\begin{abstract}
If the terms of a series behave like $n^{-k}$ where $k$ is an exactly known constant, a formula using two terms transforms the series into a series of terms like $n^{-k-2}$ provided $k \neq 1$. The multıple use of this transformation is demonstrated in summing three series.
\end{abstract}

\section{Introduction}

When the $n$th term of a series is a quotient of powers or polynomials or $\gamma$-functions in $n$ and the ratio of successive terms is close to 1 the series is difficult to sum. The three main reasons for this are that a lot of terms of the series are needed, the individual terms are much smaller than the sum, and rounding off errors in a numerical calculation can be troublesome.

Two methods for transforming such series are Lubkin's [1] transformation and Wynn's [2] $\rho$-algorithm which may be used to repeatedly transform the series or its corresponding sequence into more rapidly convergent forms.

The following method bears a close resemblance to an improvement on Wynn's $\rho$-algorithm and is easier to use.

\section{Derivation of the formula}

Let $u_{n}$ be the $n$th term of a series.

Let $Z_{n}$ be the sum to infinity of the series starting with $u_{n}$, thus $Z_{n}=u_{n}+u_{n+1}+u_{n+2}+\cdots$.

We first take a special series for which

$$
Z_{n}=\frac{A}{(n-k)(n-k+1) \cdots(n-2)} .
$$


Hence

$$
u_{n}=\frac{A(k-1)}{(n-k)(n-k+1) \cdots(n-1)}
$$

and

$$
Z_{n}=\frac{k}{k-1} \frac{u_{n-1} u_{n}}{u_{n-1}-u_{n}}=u_{n}+\frac{k}{k-1} \frac{u_{n} u_{n+1}}{u_{n}-u_{n+1}}
$$

The particular series given above is summed exactly from a knowledge of $k$ and the first two terms of the series. However if we have some other series for which $A n^{-k}$ is the dominant part of the $n$th term we may still transform the series as follows:

Let $Z_{1}=u_{1}+u_{2}+u_{3}+\cdots$ and take $u_{0}=0$ and define

$$
T_{n}=\frac{k}{k-1} \frac{u_{n-1} u_{n}}{u_{n-1}-u_{n}} \text { so } T_{1}=0
$$

then by simple rearrangements we get

$$
Z_{1}=u_{1}^{*}+u_{2}^{*}+u_{3}^{*}+\cdots
$$

where

$$
u_{n}^{*}=u_{n}-T_{n}+T_{n+1}
$$

provided that $k \neq 1$ and $\lim _{n \rightarrow \infty} T_{n}=0$.

Also if $u_{n} \sim A n^{-k}$ we substitute in equations (2) and (3) to get

$$
u_{n}^{*} \sim-[A(k+1) / 12](n-1)^{-k-2}
$$

for $n>1$ and $k \neq 1$.

The transformed series may be transformed a second time, but now the series behaves like $\sum n^{-k-2}$ so we change $k$ to $(k+2)$ in equation $(2)$ and continue to increase $k$ by 2 for each successive transformation.

Several comments on the transformation formula are:

(i) Equations (2) and (3) can be written into a calculator programme which stores $k$ and calculates $u_{n}^{*}$ when $u_{n+1}$ is inserted.

(ii) Rounding off errors are magnified because of the factor $\left(u_{n-1}-u_{n}\right)$ in the denominator of $T_{n}$ so where possible it is advisable to do at least the first transformation of the series exactly and the early transformations as accurately as possible.

(iii) $k$ may be estimated using three terms of the series. Thus when the equations (1) are approximately true we eliminate $Z_{n}$ from them to obtain an approximator 


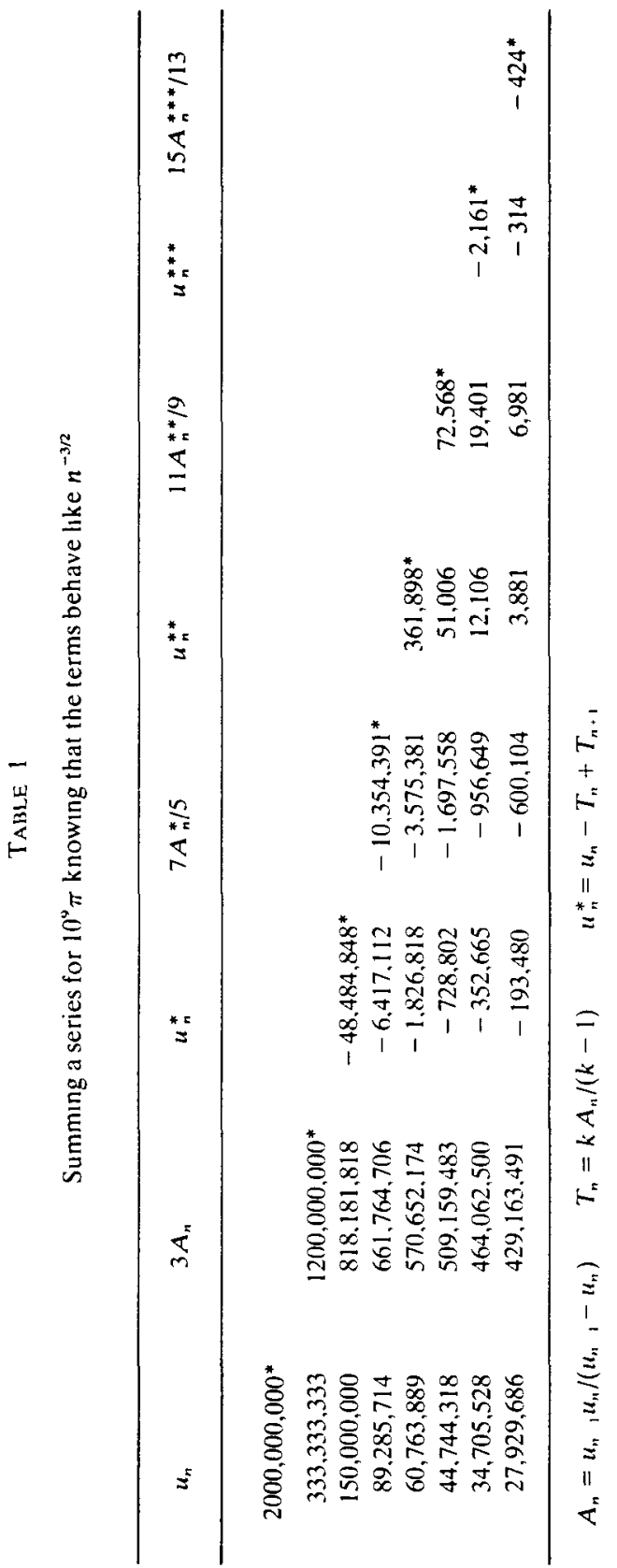




$$
k=\frac{\left(u_{n-1}-u_{n}\right)\left(u_{n}-u_{n+1}\right)}{u_{n-1} u_{n+1}-u_{n}^{2}} .
$$

(iv) If $k$ is unknown it may be eliminated from equations (1) to give Lubkin's [1] formula (17) namely

$$
Z_{n}=\frac{u_{n}\left(1-R_{n+1}\right)}{1-2 R_{n+1}+R_{n} R_{n+1}} \quad \text { where } \quad R_{n}=\frac{u_{n}}{u_{n-1}} .
$$

\section{Demonstration of the formula}

The use of equations (2) and (3) is demonstrated in table 1 on the first eight terms of a slowly convergent series for $\pi$ namely

$$
\pi=\sum_{n=0}^{\infty} \frac{(2 n) !}{2^{2 n-1}(n !)^{2}(2 n+1)}
$$

multiplied by $10^{9}$ to avoid tabulating decimals. This series would require about $10^{16}$ terms to give an error of 10 in the tabulated values or $10^{-8}$ in $\pi$.

If it is known that the first, third, fifth and seventh columns are terms of series which behave like $n^{-3 / 2}, n^{-7 / 2}, n^{-11 / 2}, n^{-15 / 2}$ then we take $k=3 / 2,7 / 2$, $11 / 2,15 / 2$ in successive uses of equation (2) or use the equivalent formulae at the bottom of table 1 in order to construct the table. The numbers marked with an asterisk at the top of each column are summed to give $3,141,592,641$ which differs from $10^{4} \pi$ by 12 .

\section{Comparison with other formulae}

The two transformations most closely related to this are those of Lubkin and Wynn.

If we do not know $k$ we may use Lubkin's transformation, the difference being that we use three terms of the series to reduce the size of the terms by a factor $(k+1) / 6(k-1) n^{2}$ instead of using two terms and a knowledge of $k$ to reduce the size of the terms by a factor $(k+1) / 12 n^{2}$. The results of summing six terms of three series for which $k$ is $1.5,2$, and 4 are given in table 2 . A comparison of columns $\mathrm{A}$ and $\mathrm{C}$ demonstrates the improvement resulting from a knowledge of $k$.

Wynn's [2] $\rho$-algorithm for transforming a sequence at the start of his section 2 is

$$
\rho_{s}\left(S_{n}\right)=\rho_{s-2}\left(S_{n+1}\right)+\frac{s}{\rho_{s-1}\left(S_{n+1}\right)-\rho_{s-1}\left(S_{n}\right)}
$$


TABLE 2

Comparison of formulae

I Six terms of $\Sigma 2(2 n) ! / 2^{2 n}(n !)^{2}(2 n+1)$

Sum

1st transformation

2nd transformation

3rd transformation

Infinite sum
A

B

$2.6781273 \quad 2.6781273$

$3.1425424 \quad 3.1425424$

$3.1415737 \quad 3.1415715$

$3.1415952 \quad 3.1415967$

$\pi=3.1415927$

II Six terms of $\Sigma n^{-2}$

A B C

$\begin{array}{lllc}\text { Sum } & 1.4913889 & 1.4913889 & 1.4913889 \\ \text { 1st transformation } & 1.6454293 & 1.6454293 & 1.6436111 \\ \text { 2nd transformation } & 1.6449244 & 1.6449226 & 1.6448949 \\ \text { 3rd transformation } & 1.6449350 & 1.6449357 & - \\ \text { Infinite sum } & & \pi^{2} / 6=1.6449341\end{array}$

III Six terms of $\sum^{-4}$

A $\quad$ B $\quad$ C

$\begin{array}{lllc}\text { Sum } & 1.08112353 & 1.08112353 & 1.08112353 \\ \text { 1st transformation } & 1.08233901 & 1.08233901 & 1.08230690 \\ \text { 2nd transformation } & 1.08232242 & 1.08232208 & 1.08232213 \\ \text { 3rd transformation } & 1.08232340 & 1.08232368 & - \\ \text { Infinite sum } & & \pi^{4} / 90=1.08232323\end{array}$

A Present formula, B Modified Wynn's $\rho$-algorithm, C Lubkin's formula.

where $S_{n}$ is the sum of a series from $u_{0}$ to $u_{n}$ inclusive and $\rho_{s}$ for even $s$ is an estimate of the sum of the infinite series. His initial conditions are ideal if $k=2$ and his three examples are of this type. However if $k$ is known and is not 2 we can take as initial conditions $\rho_{k-3}\left(S_{n}\right)=0, \rho_{k-2}\left(S_{n}\right)=S_{n}$ hence $\rho_{k-1}\left(S_{n}\right)=(k-1) / u_{n+1}$ and

$$
\rho_{k}\left(S_{n}\right)=S_{n+1}+\frac{k}{k-1} \frac{u_{n+1} u_{n+2}}{u_{n+1}-u_{n+2}} .
$$


The first two steps in this modification of Wynn's formula are now identical with the transformation derived in section 2 but thereafter the two transformations give slightly different results. In the examples in table 2 a comparison of columns $\mathrm{A}$ and $\mathrm{B}$ shows that the present formula gives results slightly closer to the infinite sum than does the $\rho$-algorithm. Also the amount of calculation done using equation (4) twice or equations (2) and (3) is comparable but in using a programmable desk calculator where most of the time taken is in inserting numbers the $\rho$-algorithm is longer.

\section{References}

[1] S. Lubkin, 'A method of summing infinite series', J. Res. Nat. Bur. Stand. 48 (1952), 228-254.

[2] P. Wynn. 'On a procrustean technique for the numerical transformation of slowly convergent sequences and serıes', Proc. Cambridge Phil. Soc. 52 (1956), 663-671.

Department of Applied Mathematics, School of General Studies, Australian National University, Canberra, A.C.T. 2600, Australia. 